\title{
Proposed therapeutic strategy for adult low-grade glioma based on aggressive tumor resection
}

\author{
Masayuki Nitta, MD, PhD, 1,2 Yoshihiro Muragaki, MD, PhD, 1,2 Takashi Maruyama, MD, PhD, 1,2 \\ Soko Ikuta, PhD, ${ }^{2}$ Takashi Komori, MD, PhD, ${ }^{3}$ Katsuya Maebayashi, MD, PhD, ${ }^{4}$ \\ Hiroshi Iseki, MD, PhD, ${ }^{2}$ Manabu Tamura, MD, PhD, ${ }^{2}$ Taiichi Saito, MD, PhD, ${ }^{1}$ Saori Okamoto, MD, ${ }^{1}$ \\ Mikhail Chernov, MD, DMedSci, ${ }^{2}$ Motohiro Hayashi, MD, PhD, ${ }^{1}$ and Yoshikazu Okada, MD, PhD ${ }^{1}$
}

\begin{abstract}
${ }^{1}$ Department of Neurosurgery; ${ }^{2}$ Faculty of Advanced Techno-Surgery (FATS), Institute of Advanced Biomedical Engineering \& Science; ${ }^{4}$ Department of Radiation Oncology, Graduate School of Medicine, Tokyo Women's Medical University; and 32Department of Pathology, Tokyo Metropolitan Neurological Hospital, Tokyo, Japan
\end{abstract}

\begin{abstract}
OBJECT There is no standard therapeutic strategy for low-grade glioma (LGG). The authors hypothesized that adjuvant therapy might not be necessary for LGG cases in which total radiological resection was achieved. Accordingly, they established a treatment strategy based on the extent of resection (EOR) and the MIB-1 index: patients with a high EOR and low MIB-1 index were observed without postoperative treatment, whereas those with a low EOR and/or high MIB-1 index received radiotherapy (RT) and/or chemotherapy. In the present retrospective study, the authors reviewed clinical data on patients with primarily diagnosed LGGs who had been treated according to the above-mentioned strategy, and they validated the treatment policy. Given their results, they will establish a new treatment strategy for LGGs stratified by EOR, histological subtype, and molecular status.
\end{abstract}

METHODS One hundred fifty-three patients with diagnosed LGG who had undergone resection or biopsy at Tokyo Women's Medical University between January 2000 and August 2010 were analyzed. The patients consisted of 84 men and 69 women, all with ages $\geq 15$ years. A total of 146 patients underwent surgical removal of the tumor, and 7 patients underwent biopsy.

RESULTS Postoperative RT and nitrosourea-based chemotherapy were administered in 48 and 35 patients, respectively. Extent of resection was significantly associated with both overall survival (OS; $p=0.0096)$ and progression-free survival (PFS; $p=0.0007$ ) in patients with diffuse astrocytoma but not in those with oligodendroglial subtypes. Chemotherapy significantly prolonged PFS, especially in patients with oligodendroglial subtypes $(p=0.0009)$. Patients with a mutant IDH1 gene had significantly longer OS $(p=0.034)$. Multivariate analysis did not identify MIB-1 index or RT as prognostic factors, but it did identify chemotherapy as a prognostic factor for PFS and EOR as a prognostic factor for OS and PFS.

CONCLUSIONS The findings demonstrated that EOR was significantly correlated with patient survival; thus, one should aim for maximum tumor resection. In addition, patients with a higher EOR can be safely observed without adjuvant therapy. For patients with partial resection, postoperative chemotherapy should be administered for those with oligodendroglial subtypes, and repeat resection should be considered for those with astrocytic tumors. More aggressive treatment with RT and chemotherapy may be required for patients with a poor prognosis, such as those with diffuse astrocytoma, $1 p / 19 q$ nondeleted tumors, or IDH1 wild-type oligodendroglial tumors with partial resection.

http://thejns.org/doi/abs/10.3171/2014.10.FOCUS14651

KEY WORDS low-grade glioma; surgical resection; survival; therapeutic strategy; tumor subtype

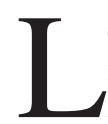

OW-GRADE glioma (LGG) is a slowly progressive yet invasive tumor that generally arises in young adults. ${ }^{11}$ However, about half of all LGG cases eventually progress to malignant transformation, and in such cases, the prognosis is dismal. ${ }^{8}$ There was no standard therapeu- tic strategy for LGG; however, the tumor is often resected with or without subsequent fractionated radiotherapy (RT) and chemotherapy using nitrosourea or temozolomide. . $^{5,15}$

Recently, the significance of the extent of resection (EOR) has been reported for $\mathrm{LGG},{ }^{12,14,18,19,25}$ and maxi-

ABBREVIATIONS ACNU = nimustine hydrochloride; EOR = extent of resection; EORTC = European Organisation for Research and Treatment of Cancer; LGG = lowgrade glioma; OS = overall survival; PCV = procarbazine, lomustine, vincristine; $P F S$ = progression-free survival; $R T=$ radiotherapy. ACCOMPANYING EDITORIAL See E8. DOI: 10.3171/2014.10.FOCUS14719.

SUBMITTED September 6, 2014. ACCEPTED October 9, 2014.

INCLUDE WHEN CITING DOI: 10.3171/2014.10.FOCUS14651.

DISCLOSURE The authors report no conflict of interest concerning the materials or methods used in this paper or the findings specified in this paper. 
mum resection has recently been proposed as the standard protocol. However, no randomized trial has examined the effect of EOR on the survival of patients with LGG. The European Organisation for Research and Treatment of Cancer (EORTC) 22845 randomized trial showed that early RT for LGG prolonged progression-free survival (PFS) but not overall survival (OS). ${ }^{22}$ Recent reports have shown that postoperative RT improved PFS but not OS in patients with LGG who had undergone partial tumor resection. ${ }^{19,25}$ Therefore, the effect of RT on the long-term survival of patients with LGG remains controversial.

Moreover, the role of chemotherapy in LGG is complicated. Although combination chemotherapy with procarbazine, lomustine, and vincristine (PCV) demonstrated promising results in the $1 \mathrm{p} / 19 \mathrm{q}$ codeleted oligodendroglial tumors ${ }^{1,3}$ in the early 2000 s, its long-term efficacy was disproved in the mid 2000s. ${ }^{2,23}$ More recent long-term results of a randomized trial (EORTC 9802) indicated both that the RT+PCV therapy prolonged PFS but not OS in patients with LGG and that this therapy may be beneficial for 2-year survivors. ${ }^{21}$ The role of chemotherapy in diffuse astrocytoma is unclear, and no controlled clinical trial has focused on diffuse astrocytoma. Recent developments in molecular analysis, especially for the $1 \mathrm{p} / 19 \mathrm{q}$ locus and the IDHI (isocitrate dehydrogenase I) gene, have allowed more convenient molecular subclassification..$^{10}$ Furthermore, the above-mentioned factors have prognostic and predictive value for several tumor subtypes. Thus, LGG treatment should be decided based on the tumor's histological and molecular characteristics.

Since 2000, our institute has established "informationguided surgery" using intraoperative MRI and initiated maximum resection for gliomas. ${ }^{6,13}$ We hypothesized that adjuvant therapy might not be necessary for LGGs if total radiological resection was achieved. Additionally, as the E3 ubiquitin-protein ligase MIB-1 labeling index is generally proportional to the malignancy of gliomas, ${ }^{24}$ we hypothesized that a LGG with a higher MIB-1 index might require postoperative therapy. Thus, we established the following treatment strategy based on EOR and the MIB1 index: patients with a high EOR and low MIB-1 index were observed without postoperative treatment, whereas those with a low EOR and/or high MIB-1 index received RT and/or chemotherapy.

In the present retrospective study, we reviewed the clinical data of patients with primarily diagnosed LGG who had been treated with the above-mentioned strategy, and we validated the treatment policy. We then established a new treatment strategy for LGG stratified by EOR, histological subtype, and molecular status.

\section{Methods}

\section{Patient Population}

One hundred fifty-three patients, including 84 males and 69 females (ages $\geq 15$ years), who had been diagnosed with LGG and had undergone resection or biopsy at Tokyo Women's Medical University between January 2000 and August 2010 were analyzed. Patients who had undergone prior resection of the tumor were excluded; however, those who had undergone previous biopsy performed as part of a diagnostic workup leading to the eventual surgical removal of the tumor at our institution were eligible for analysis. Patients with neurofibromatosis Type 1, pleomorphic xanthoastrocytoma, or infratentorial lesions were excluded from the study. Tumor grading and pathological diagnosis were performed according to the WHO guidelines. Clinical data were collected from patient records and telephone interviews. Two outcome measures were assessed: OS and PFS. Overall survival was defined as the time between initial surgery and death, whereas PFS was defined as the time between initial surgery and the demonstration of an unequivocal increase in tumor size on follow-up imaging and obvious clinical deterioration. Patients with no known progression were censored as of their last visit and/or scan date.

\section{Molecular Analysis}

Codeletion of $1 \mathrm{p} / 19 \mathrm{q}$ was analyzed using the fluorescence in situ hybridization method. Mutation of IDHI was investigated by immunohistochemical analysis of paraffin-embedded surgical specimens using anti-IDH1R132S antibody (Dianova).

\section{Treatment}

A total of 146 patients underwent surgical removal of the tumor. Maximum resection of the T2 high-signalintensity lesion was performed using an updated navigation system and intraoperative MRI. ${ }^{13}$ Seven patients underwent biopsy. Postoperative treatment was conducted as follows (Table 1): patients with an EOR $\geq 95 \%$ and an MIB-1 index < 5\% (Group A) were observed without any postoperative treatment, whereas those with an EOR < 95\% or an MIB-1 index $\geq 5 \%$ (Group B) received fractionated RT and chemotherapy with nimustine hydrochloride (ACNU) and vincristine. In 2004, we changed the therapeutic strategy for Group B, dividing the patients into two subgroups based on their $1 \mathrm{p} / 19 \mathrm{q}$ codeletion status. Patients with $1 \mathrm{p} / 19 \mathrm{q}$ codeletion received RT and chemotherapy with procarbazine, $\mathrm{ACNU}$, and vincristine (PAV), instead of PCV because lomustine has not been approved in Japan, whereas those without $1 \mathrm{p} / 19 \mathrm{q}$ codeletion received only RT. In 2007, we changed the EOR threshold from 95\% to $90 \%$ because interim analysis showed that the OS for Group A was significantly longer than for Group B (data not shown). Forty-eight patients received extended local fractionated RT (range 50-60 Gy), and 35 received nitrosourea-based chemotherapy within 5 weeks after surgical treatment; 29 of these 35 received both RT and chemotherapy.

\section{Volumetric Analysis}

Tumor volumes, as determined from axial T2-weighted MRI, were calculated by importing DICOM (Digital Imaging and Communications in Medicine) images from MRI to Leksell GammaPlan software (Elekta). Extent of resection was calculated as follows: (preoperative tumor volume - postoperative tumor volume)/preoperative tumor volume.

\section{Statistical Analysis}

Overall survival and PFS were estimated using the Kaplan-Meier method. A log-rank test was used to evalu- 
TABLE 1. Treatment strategy for LGGs in adults at a single institution in 2000-2010

\begin{tabular}{|c|c|c|c|c|}
\hline \multirow[b]{2}{*}{ Years } & \multicolumn{2}{|c|}{ Group A } & \multicolumn{2}{|l|}{ Group B } \\
\hline & Pretreatment Factors & Treatment & Pretreatment Factors & Treatment \\
\hline $2000-2003$ & EOR $\geq 95 \% \&$ MIB- $1<5 \%$ & Observation & EOR $<95 \%$ or MIB- $1 \geq 5 \%$ & $\mathrm{RT}+\mathrm{ACNU} / \mathrm{VCR}$ \\
\hline \multirow[t]{2}{*}{ 2004-2006 } & EOR $\geq 95 \% \&$ MIB- $1<5 \%$ & Observation & EOR $<95 \%$ or MIB- $1 \geq 5 \%$ \& $1 p 19 q$ codeletion (-) & RT \\
\hline & & & EOR $<95 \%$ or MIB-1 $\geq 5 \% \& 1 p 19 q$ codeletion $(+)$ & RT+PAV \\
\hline \multirow[t]{2}{*}{$2007-2010$} & EOR $\geq 90 \% \&$ MIB- $1<5 \%$ & Observation & EOR $<90 \%$ or MIB- $1 \geq 5 \%$ \& $1 p 19 q$ codeletion (-) & RT \\
\hline & & & EOR $<90 \%$ or MIB- $1 \geq 5 \%$ \& $1 p 19 q$ codeletion $(+)$ & $\mathrm{RT}+\mathrm{PAV}$ \\
\hline
\end{tabular}

PAV = procarbazine, $\mathrm{ACNU}$, vincristine; $\mathrm{VCR}=$ vincristine; $(+)=$ positive codeletion status; $(-)$ = negative codeletion status.

ate the importance of prognostic factors that may affect survival. Data analysis was performed using the JMP statistical software (SAS Inc.). Univariate analyses for OS and PFS were performed using Cox proportional-hazards modeling. Variables that were statistically significant or showed borderline significance on univariate analysis were further analyzed with multivariate analysis using Cox proportional-hazards modeling. Hazard ratios and 95\% confidence intervals are reported with the 2-tailed probability values. The reported probability values in the Cox model are based on the Wald test, and values $<0.05$ were considered significant.

\section{Results}

The median age was 37.0 years (range 15-76 years), and the median preoperative Karnofsky Performance Scale score was 100 (range 70-100). Forty-nine patients (32.0\%) patients had diffuse astrocytoma, 45 (29.4\%) had oligoastrocytoma, and $59(38.6 \%)$ had oligodendroglioma. The median MIB-1 labeling index was $4.2 \%$ (range $0.3 \%-$ $21.0 \%$ ). The median time to progression was 7.4 years. Mutation of IDHI (R132S) was found in 111 (75.0\%) of 148 patients analyzed. Most of the oligodendroglial subtypes and about half of the astrocytic tumors had $I D H 1$ mutation: 34 (79.1\%) of 43 oligoastrocytomas, $52(88.1 \%)$ of 59 oligodendrogliomas, and 25 (54.3\%) of 46 diffuse astrocytomas. Codeletion of $1 \mathrm{p} / 19 \mathrm{q}$ was detected in 63 $(56.8 \%)$ of 111 patients investigated, including $4(15.4 \%)$ of 26 with diffuse astrocytomas, $21(60.0 \%)$ of 35 with oligoastrocytomas, and $38(76.0 \%)$ of 50 with oligodendrogliomas. The 5-year and 10-year OS rates for all patients were $95.1 \%$ and $85.4 \%$, respectively. ${ }^{14}$

\section{Extent of Resection and Prognosis}

As reported in our previous paper, both OS and PFS were significantly longer in patients with $\geq 90 \%$ EOR (Fig. 1A and B). ${ }^{14}$ When patients were divided into the subgroups of diffuse astrocytoma and oligodendroglial subtypes, EOR was significantly associated with both OS $(\mathrm{p}=0.0096)$ and PFS $(\mathrm{p}=0.0007)$ in patients with diffuse astrocytoma (Fig. 1C and D). On the other hand, EOR did not affect OS and PFS in patients with oligodendroglial subtypes (Fig. 1E and F).

\section{Radiotherapy and Prognosis}

Postoperative RT was performed in 48 patients, includ- ing $19(38.8 \%)$ of 49 with diffuse astrocytomas, 18 (40.0\%) of 45 with oligoastrocytomas, and 11 (18.6\%) of 59 with oligodendrogliomas. Radiotherapy did not correlate with OS $(\mathrm{p}=0.054)$ and PFS $(\mathrm{p}=0.69)$ in the analysis of all patients (Fig. 2A and B), and subgroup analysis did not show a survival benefit with RT in patients with either diffuse astrocytoma or oligodendroglial subtypes (Fig. 2C-F). Patients with oligodendroglial subtypes who received postoperative RT had longer PFS ( $p=0.02)$, whereas those with diffuse astrocytoma who received RT exhibited a trend toward shorter PFS, although the relationship did not reach statistical significance. However, the effect of RT on oligodendroglial subtypes might be confounded by chemotherapy, because chemotherapy, not RT, was identified as a prognostic factor for PFS on multivariate analysis (Table 2).

\section{Chemotherapy and Prognosis}

Postoperative chemotherapy was administered in 35 patients, including 9 (18.4\%) of 49 with diffuse astrocytomas, $16(35.6 \%)$ of 45 with oligoastrocytomas, and 10 (16.9\%) of 59 with oligodendrogliomas. In the analysis of all patients, chemotherapy significantly prolonged PFS ( $p$ $=0.01)$, but there was no correlation with $\mathrm{OS}(\mathrm{p}=0.5$; Fig. 3A and B). Thus, a longer follow-up period might be required to elucidate the role of chemotherapy in patient survival. There was no association between chemotherapy and either OS $(\mathrm{p}=0.2)$ or PFS $(\mathrm{p}=0.8)$ in patients with diffuse astrocytoma (Fig. 3C and D), whereas chemotherapy was strongly associated with PFS $(p=0.0009)$ in patients with oligodendroglial subtypes (Fig. 3F). As there were only a few deaths among the patients with oligodendroglial subtypes, it was difficult to clarify the effect of chemotherapy on OS (Fig. 3E). Therefore, our findings recommend chemotherapy in patients with oligodendroglial subtypes who undergo partial tumor resection. On the other hand, repeated surgery aiming at additional resection may be necessary in patients with diffuse astrocytoma whose EOR was low in the first surgery.

\section{Molecular Status and Prognosis}

Patients with codeletion of $1 \mathrm{p} / 19 \mathrm{q}$ locus showed significantly longer PFS $(\mathrm{p}=0.0048)$, but no OS differences $(\mathrm{p}=$ 0.4 ) were observed between patients with and without such a deletion, probably because of the small number of death events (Fig. 4A and B). Chemotherapy significantly prolonged PFS in patients with oligodendroglial subtypes regardless of their $1 p / 19 q$ codeletion status (data not shown). 

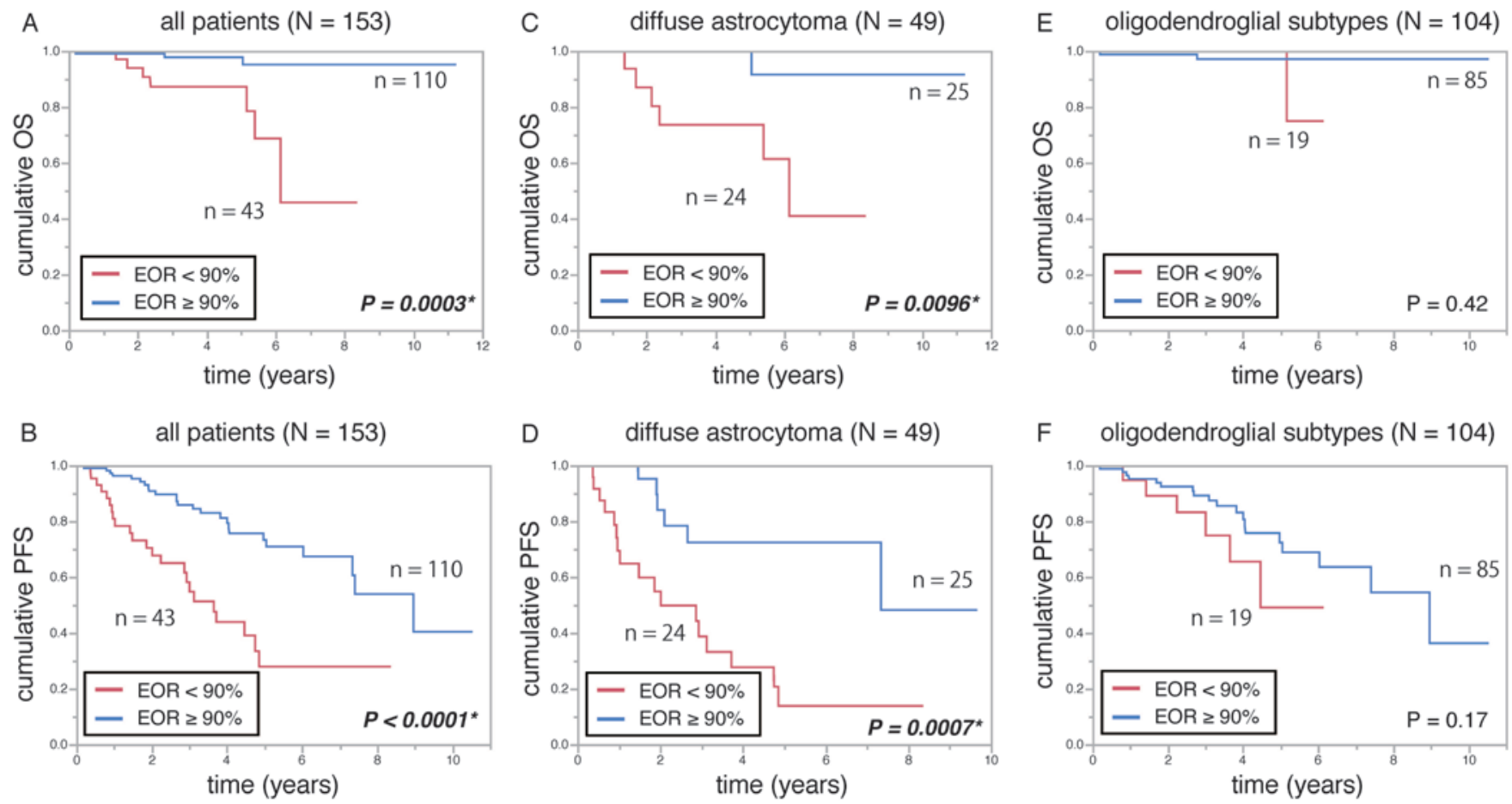

FIG. 1. Overall survival (A) and PFS (B) for all patients, according to EOR. Overall survival (C) and PFS (D) for patients with diffuse astrocytoma, according to EOR. Overall survival $(E)$ and PFS (F) for patients with oligodendroglial subtypes, according to EOR. $N$ or $n=$ number of patients. Asterisks indicate significant $p$ values.

In the analysis of all patients, IDHI status was significantly associated with OS ( $\mathrm{p}=0.034)$ but not with PFS (p $=0.12$; Fig. $4 \mathrm{C}$ and D). In a subgroup analysis, $I D H 1$ status did not correlate with either OS or PFS in patients with diffuse astrocytoma (data not shown).

Interestingly, EOR was strongly associated with OS in
A all cases $(\mathrm{N}=153)$

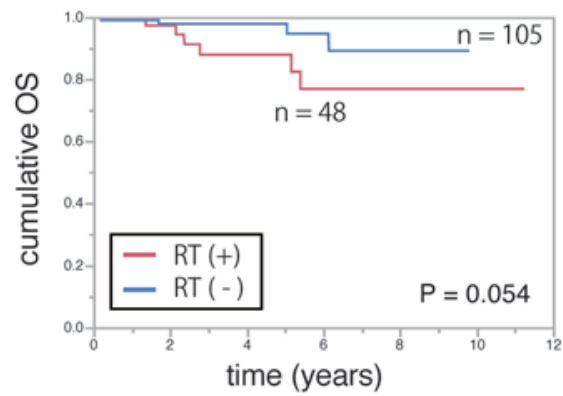

B

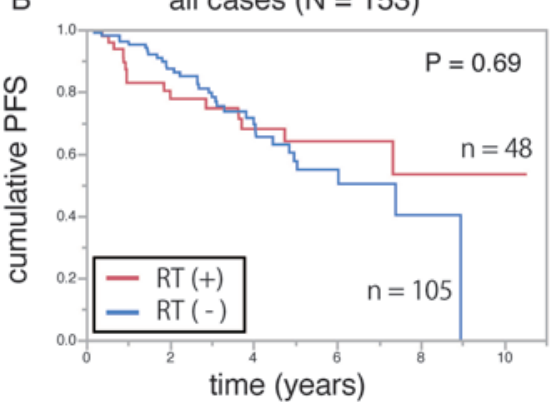

C diffuse astrocytoma $(\mathrm{N}=49)$

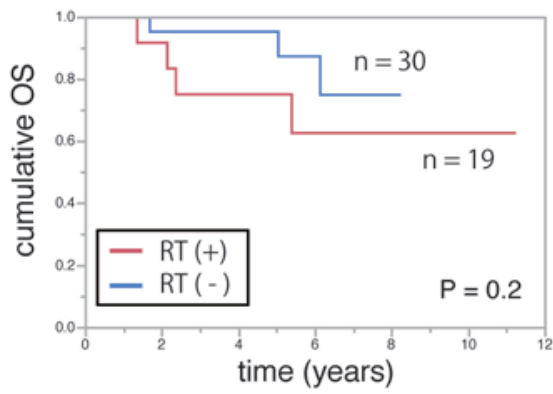

D diffuse astrocytoma $(\mathrm{N}=49)$

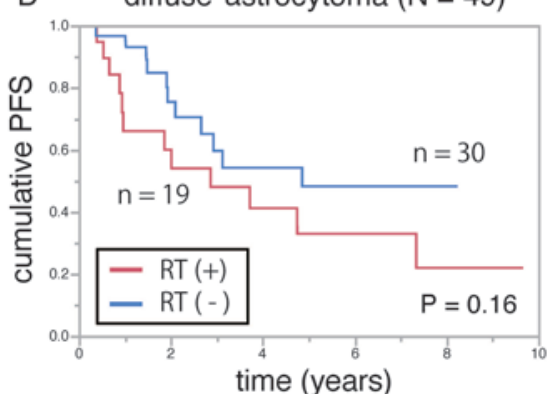

E oligodendroglial subtypes $(\mathrm{N}=104)$

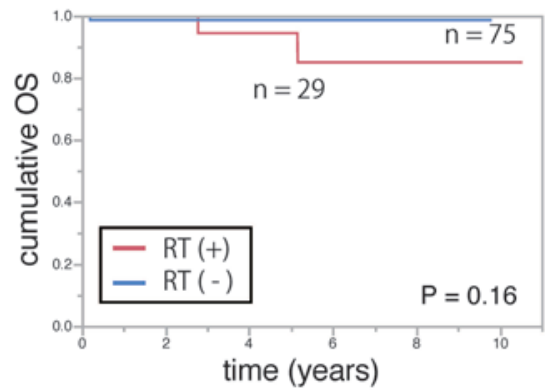

F oligodendroglial subtypes $(\mathrm{N}=104)$

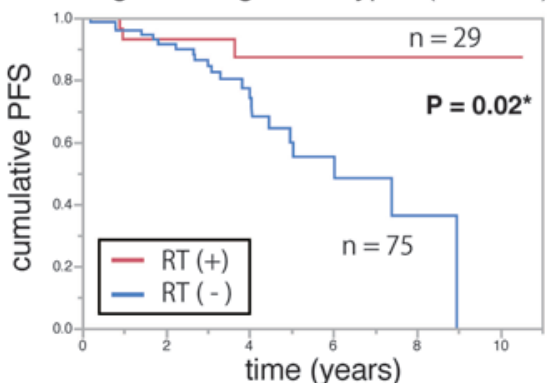

FIG. 2. Overall survival (A) and PFS (B) for all patients, according to treatment with $(+)$ or without (-) RT. Overall survival (C) and PFS (D) for patients with diffuse astrocytoma, according to treatment with or without RT. Overall survival (E) and PFS (F) for patients with oligodendroglial subtypes, according to treatment with or without RT. 
TABLE 2. Results of univariate and multivariate analysis

\begin{tabular}{|c|c|c|c|c|c|c|}
\hline \multirow[b]{2}{*}{ Factor } & \multicolumn{3}{|c|}{ Univariate Analysis } & \multicolumn{3}{|c|}{ Multivariate Analysis } \\
\hline & $\mathrm{HR}$ & $95 \% \mathrm{Cl}$ & $p$ Value & $\mathrm{HR}$ & $95 \% \mathrm{Cl}$ & $p$ Value \\
\hline \multicolumn{7}{|l|}{ OS } \\
\hline Age in yrs: $\geq 50$ vs < $<0$ & 5.43 & $1.55-21.30$ & $0.0089^{*}$ & 5.38 & $1.46-22.10$ & $0.0121^{*}$ \\
\hline Tumor diameter in $\mathrm{cm}: \geq 5$ vs $<5$ & 1.52 & $0.43-5.97$ & 0.5087 & 1.08 & $0.29-4.43$ & 0.9026 \\
\hline Tumor subtype: DA vs O/OA & 4.98 & $1.37-23.27$ & $0.0143^{*}$ & 5.23 & $1.33-26.08$ & $0.0172^{*}$ \\
\hline RT: no vs yes & 3.30 & $0.07-1.08$ & 0.0656 & 0.34 & $0.06-1.97$ & 0.2234 \\
\hline Chemotherapy: no vs yes & 0.68 & $0.19-2.70$ & 0.5667 & 0.99 & $0.14-4.95$ & 0.8978 \\
\hline \multicolumn{7}{|l|}{ PFS } \\
\hline Age (yrs): $\geq 50$ vs $<50$ & 1.58 & $0.80-2.93$ & 0.1757 & 1.41 & $0.70-2.67$ & 0.3149 \\
\hline Tumor diameter in $\mathrm{cm}: \geq 5$ vs $<5$ & 1.59 & $0.89-2.89$ & 0.1122 & 1.89 & $1.02-3.55$ & $0.0428^{*}$ \\
\hline Tumor subtype: DA vs O/OA & 2.08 & $1.16-3.72$ & $0.0140^{*}$ & 1.86 & $1.00-3.40$ & $0.0485^{\star}$ \\
\hline RT: no vs yes & 1.1 & $0.60-2.12$ & 0.7422 & 0.73 & $0.33-1.72$ & 0.463 \\
\hline Chemotherapy: no vs yes & 2.27 & $1.13-5.07$ & $0.0195^{*}$ & 3.17 & $1.23-6.56$ & $0.0161^{*}$ \\
\hline
\end{tabular}

$\mathrm{DA}=$ diffuse astrocytoma; $\mathrm{O}$ = oligodendroglioma; $\mathrm{OA}$ = oligoastrocytoma.

* Statistically significant.

patients with wild-type $I D H I$, although it did not affect OS in patients with IDHI mutation (data not shown). Thus, our results indicated extensive resection for patients without IDHI mutation. However, chemotherapy significantly prevented recurrence in patients with mutant IDHI (p < $0.0001)$ but did not affect PFS ( $p=0.051)$ in those with wild-type IDHI (Fig. 4E and F). Thus, our findings suggest that repeat surgery should be considered for patients with wild-type $I D H I$ who have partial tumor removal in their first operation.

\section{Univariate and Multivariate Analysis}

In this study, we examined univariate and multivariate modeling using the following parameters: patient age, tumor diameter, tumor subtype, RT, and chemotherapy. Among these factors, multivariate analysis identified tumor subtype and chemotherapy for PFS, suggesting that the role of chemotherapy in preventing a recurrence depends on the tumor subtype (Table 2). However, the parameters identified as significant for OS were the conventional risk factors of age and tumor subtype, but not chemotherapy.
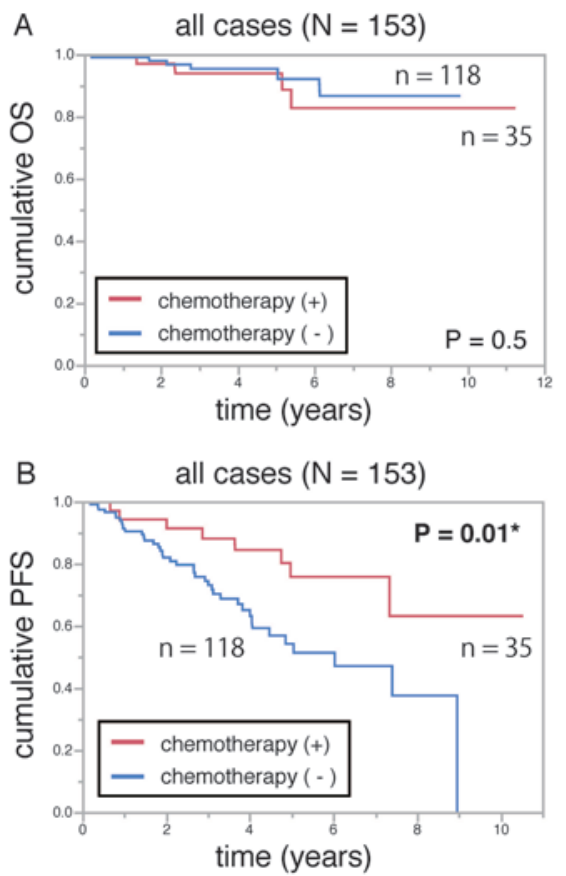

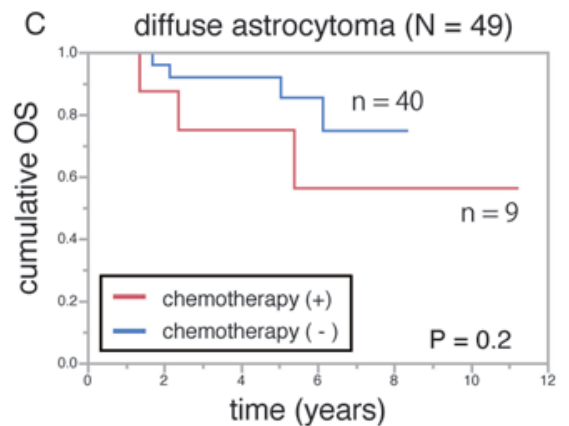

D diffuse astrocytoma $(\mathrm{N}=49)$

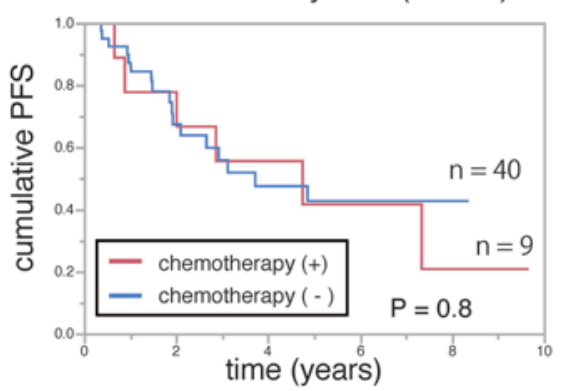

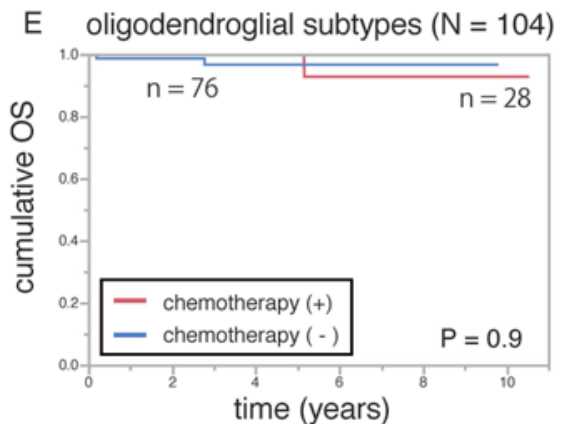

F oligodendroglial subtypes $(\mathrm{N}=104)$

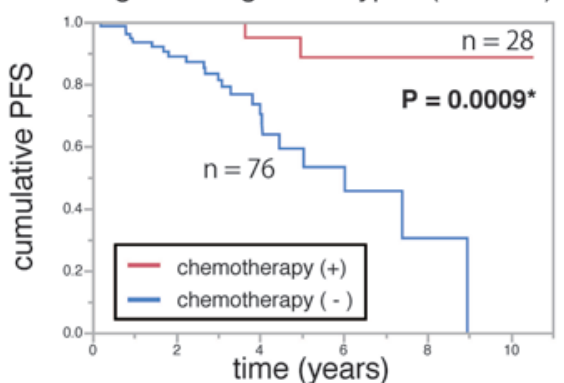

FIG. 3. Overall survival (A) and PFS (B) for all patients, according to treatment with or without chemotherapy. Overall survival (C) and PFS (D) for patients with diffuse astrocytoma, according to treatment with or without chemotherapy. Overall survival (E) and PFS (F) for patients with oligodendroglial subtypes, according to treatment with or without chemotherapy. 

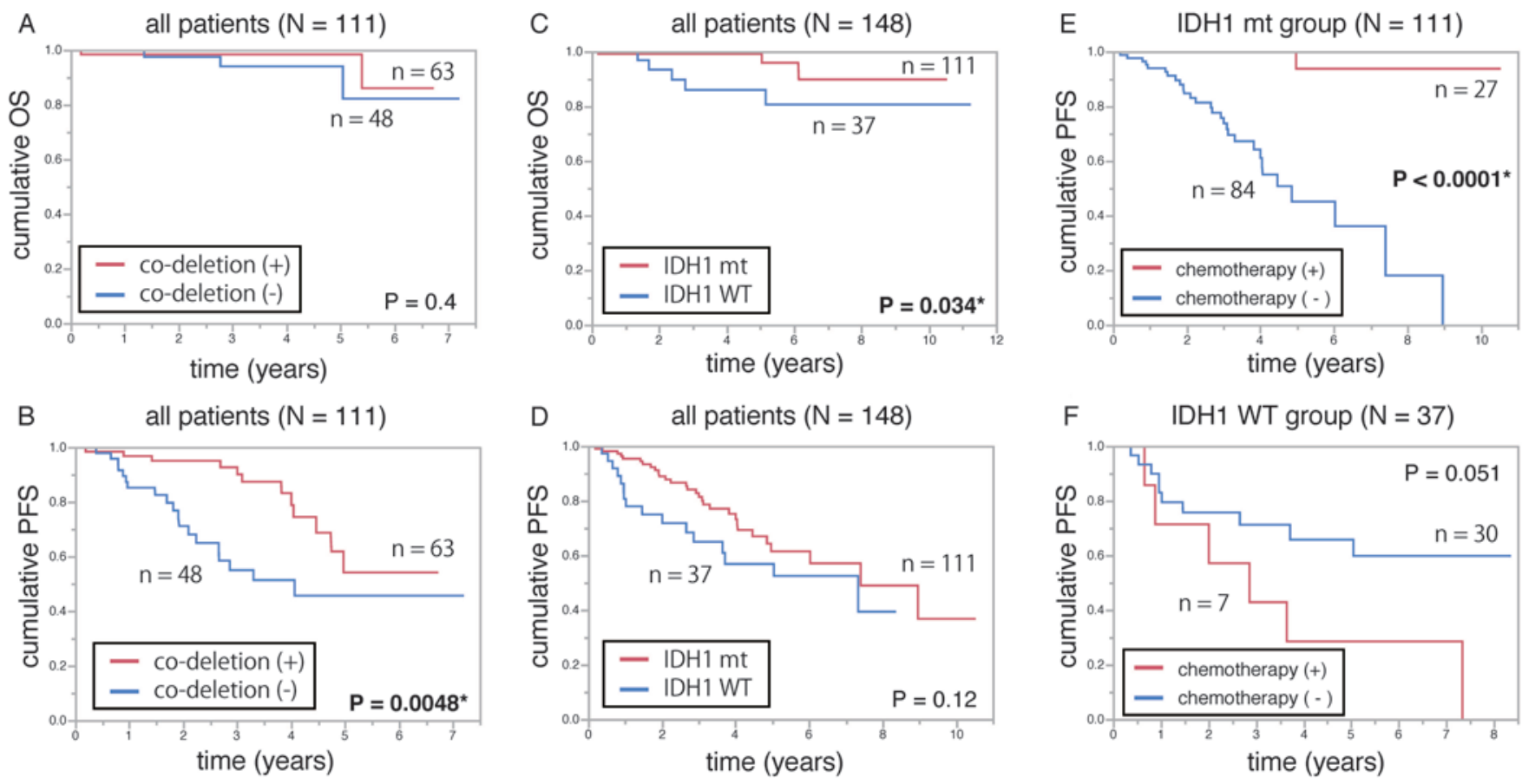

FIG. 4. Overall survival (A) and PFS (B) for all patients, according to status of $1 p / 19 q$ codeletion. Overall survival (C) and PFS (D) for all patients, according to status of IDH1 mutation. Progression-free survival for patients with mutant (mt) IDH1 (E) and wild-type (WT) IDH1 (F), according to treatment with or without chemotherapy.

When EOR was added as a candidate factor for multivariate analysis, only EOR was selected for PFS, whereas EOR and age were selected for OS. ${ }^{14}$ Tumor subtype was not selected in the analysis including EOR, suggesting that EOR is a strong prognostic factor regardless of tumor subtypes. The limitation of the multivariate analysis in this study is the confounding bias between EOR and RT/chemotherapy, because patients with a high EOR did not receive RT or chemotherapy and these factors cannot be included at the same time.

\section{Discussion}

\section{New Treatment Strategy for LGG}

In our previous report, we showed that EOR was strongly associated with OS in patients with LGG but that the MIB-1 index was not associated with OS in these patients. ${ }^{14}$ In the present study, the associations of RT, chemotherapy, and molecular status with patient survival were analyzed in detail. Given our results, we will update our treatment strategy for LGG, including 3 major changes (Fig. 5). First, the MIB-1 index will be excluded because none of the analyses showed statistical differences in patient survival according to this index. Second, patients with a low EOR (Group B) will be further divided into 2 subgroups according to histological subtypes, as there were significant differences in the response to chemotherapy between astrocytic and oligodendroglial tumors. Finally, treatment of patients with oligodendroglial subtypes will be stratified into 2 categories as follows: chemotherapy only for patients with $1 \mathrm{p} / 19 \mathrm{q}$ codeletion and/or mutant $I D H 1$, and RT and chemotherapy for those who had neither $1 \mathrm{p} / 19 \mathrm{q}$ codeletion nor an IDHI mutation.
This stratification was performed given the worse prognosis in the latter group.

In our prospective case series, we stratified postoperative treatment by EOR instead of age. Age is among the previously described risk factors for a poor prognosis in cases of LGG. ${ }^{16}$ As a result, patients with an EOR $\geq 90 \%$ achieved excellent clinical courses regardless of their age, with 5- and 10-year OS rates of $97.8 \%$ and $95.3 \%$, respectively. Although another clinical trial stratified adjuvant therapy by EOR and age, ${ }^{21}$ our results strongly suggested that patients with a high EOR could be safely observed without postoperative therapy and that maximum tumor resection should be aimed for in LGG. However, EOR did not affect OS and PFS in patients with oligodendroglial subtypes (Fig. 1E and F). The underlying explanation for this result might be that chemotherapy was largely effective in preventing recurrences of oligodendroglial tumors (Fig. 3F). Further, patients with oligodendroglial subtypes who received chemotherapy had a low EOR.

In this study, chemotherapy significantly prolonged PFS according to both univariate and multivariate analyses (Table 2). Subgroup analyses based on histological subtypes revealed that the correlation between chemotherapy and PFS was significant in patients with oligodendroglial subtypes, but not in patients with diffuse astrocytoma (Fig. 3). Thus, when EOR is less than $90 \%$, chemotherapy should be recommended for oligodendroglial subtypes, but repeat resection should be considered in patients with diffuse astrocytoma. A good example of such a scenario involves cases of LGG located in an eloquent area with biopsy or partial resection performed at another institution. It is possible to perform repeated sur- 


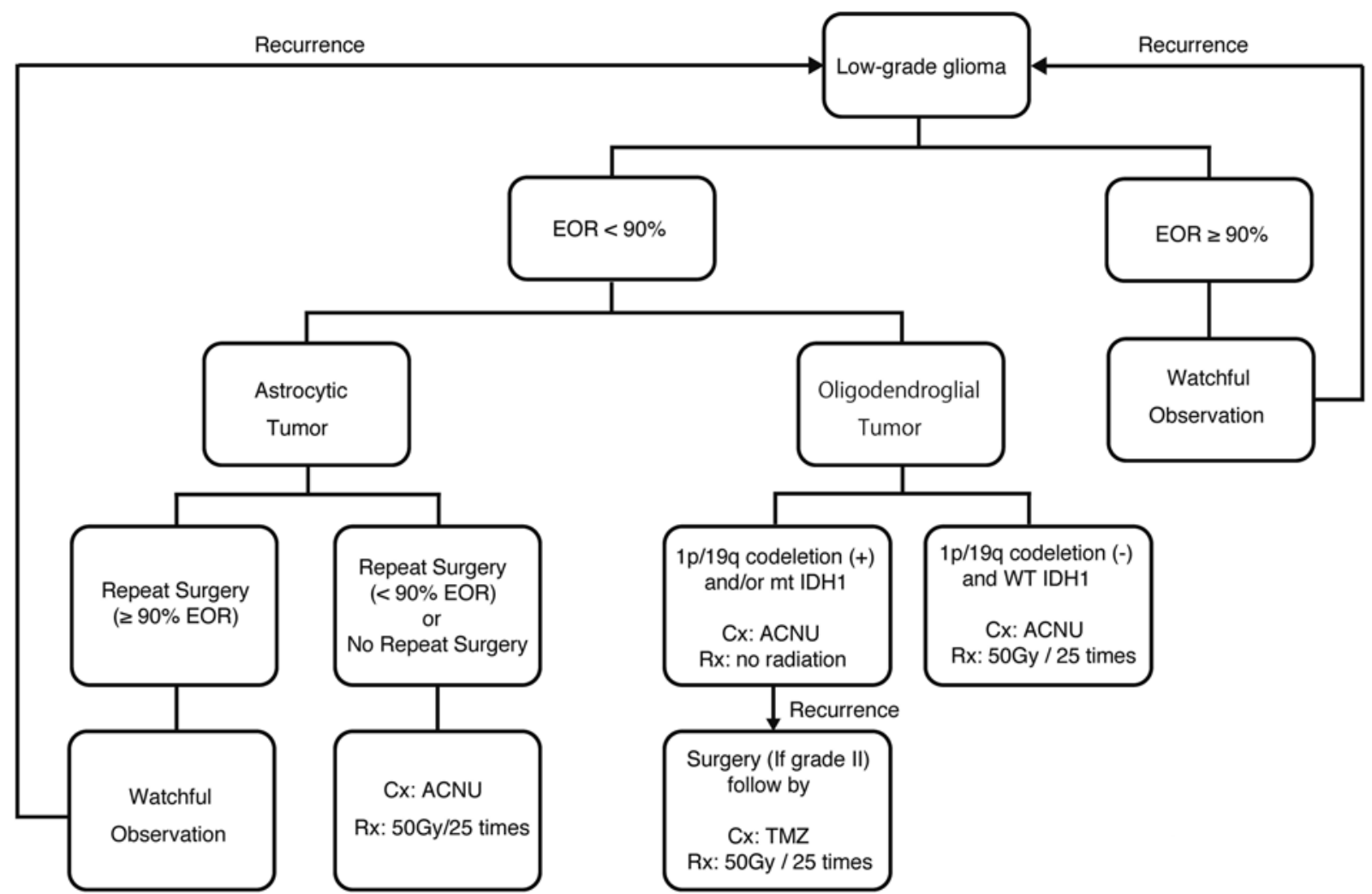

FIG. 5. Therapeutic strategy for LGG stratified by EOR, histological subtype, and molecular status. Patients with $\geq 90 \%$ EOR can be safely observed regardless of their tumor subtype. Patients with $<90 \%$ EOR are divided into two groups, diffuse astrocytoma and oligodendroglial subtypes, and those with diffuse astrocytoma are recommended for repeat surgery, whereas those with oligodendroglial subtypes are treated with chemotherapy with or without RT according to their molecular status. $\mathrm{Cx}=$ chemotherapy; $\mathrm{Rx}=$ radiation; TMZ = temozolomide. Modified from Nitta et al: Neurol Med Chir (Tokyo) 53:447-454, 2013. Published with permission.

gery using awake craniotomy to achieve maximum tumor resection for these patients. ${ }^{4}$ Furthermore, it is noteworthy that chemotherapy significantly prolonged PFS in patients with mutant $I D H I(\mathrm{p}<0.0001)$. In contrast, patients with wild-type IDHI (mostly in cases of the astrocytic subtype) who had received chemotherapy showed a poorer prognosis than those who did not receive chemotherapy (Fig. 4). This result supports the notions that 1) patients with diffuse astrocytoma harboring wild-type $I D H I$ would not benefit from chemotherapy and 2) tumor resection has the strongest impact on astrocytic tumors.

Our multivariate analysis did not identify any significant role for RT, whereas PFS was prolonged among patients with oligodendroglial subtypes who had received RT (Fig. 2F). Since most of the patients who had received RT also received chemotherapy, this effect might be attributable to chemotherapy. Radiotherapy has been routinely administered for LGG, and the appropriate RT dosages were investigated in the 1980s and 1990s. ${ }^{7,20}$ However, higher doses of fractionated RT for LGG did not show a survival benefit. The EORTC 22845 trials reported that early RT did not affect $\mathrm{OS},{ }^{22}$ and recent retrospective analyses showed that postoperative RT did not prolong OS..$^{19,25}$ On the other hand, Pouratian et al. showed improved outcomes for older patients receiving postoperative RT, ${ }^{17}$ and Youland et al. reported that patients in the 1980s and 1990s in whom EOR was lower may have benefited from
RT. ${ }^{25}$ Thus, there might be a subgroup that benefits from postoperative RT. Therefore, a randomized clinical trial is necessary to clarify the role of postoperative RT. In our new strategy, RT is only administered to patients with a poor prognosis, such as those who have diffuse astrocytoma with a low EOR or those who have oligodendroglial tumors with a low EOR, without $1 \mathrm{p} / 19 \mathrm{q}$ codeletion, and without IDHI mutation. Although it is reasonable to provide stronger treatment to patients with a poor prognosis, it is debatable whether both RT and chemotherapy should be administered in patients with poor-prognosis LGG. The definitive roles of RT and chemotherapy have been demonstrated in patients with anaplastic gliomas; therefore, these treatments might be more effective after malignant transformation. New therapeutic strategies might be required for patients with a poor prognosis.

This study has limitations. First, although we performed a volumetric analysis, accurate estimation of the EOR is difficult for diffuse tumors that have unclear borders. Second, since patients with a low EOR received adjuvant therapy in this study, there is strong confounding between EOR and RT/chemotherapy. Thus, it is difficult to accurately evaluate the role of RT and chemotherapy. Third, a number of patients received both RT and chemotherapy. Therefore, it is difficult to accurately compare the roles of RT and chemotherapy alone. Therefore, a controlled clinical trial will be necessary in the future. 


\section{Conclusions}

The clinical management of LGG has not been standardized. Our findings demonstrated that EOR significantly correlated with patient survival; thus, we should aim for maximum tumor resection. In addition, patients with a higher EOR can be safely observed without adjuvant therapy. For those with partial resection, postoperative chemotherapy should be administered and repeat resection should be considered for those with astrocytic tumor. Patients with a poor prognosis, such as those having diffuse astrocytoma and $1 \mathrm{p} / 19 \mathrm{q}$ nondeleted or $I D H 1$ wild-type oligodendroglial tumors with partial resection, may require more aggressive treatment with RT and chemotherapy.

\section{References}

1. Bauman GS, Ino Y, Ueki K, Zlatescu MC, Fisher BJ, Macdonald DR, et al: Allelic loss of chromosome 1p and radiotherapy plus chemotherapy in patients with oligodendrogliomas. Int J Radiat Oncol Biol Phys 48:825-830, 2000

2. Cairncross G, Berkey B, Shaw E, Jenkins R, Scheithauer B, Brachman D, et al: Phase III trial of chemotherapy plus radiotherapy compared with radiotherapy alone for pure and mixed anaplastic oligodendroglioma: Intergroup Radiation Therapy Oncology Group Trial 9402. J Clin Oncol 24:2707-2714, 2006

3. Cairncross JG, Ueki K, Zlatescu MC, Lisle DK, Finkelstein DM, Hammond RR, et al: Specific genetic predictors of chemotherapeutic response and survival in patients with anaplastic oligodendrogliomas. J Natl Cancer Inst 90:1473-1479, 1998

4. De Benedictis A, Moritz-Gasser S, Duffau H: Awake mapping optimizes the extent of resection for low-grade gliomas in eloquent areas. Neurosurgery 66:1074-1084, 2010

5. Hafeez S, Cavaliere R: Recent innovations in the management of low-grade gliomas. Curr Treat Options Neurol 14:369-380, 2012

6. Iseki H, Nakamura R, Muragaki Y, Suzuki T, Chernov M, Hori T, et al: Advanced computer-aided intraoperative technologies for information-guided surgical management of gliomas: Tokyo Women's Medical University experience. Minim Invasive Neurosurg 51:285-291, 2008

7. Karim AB, Maat B, Hatlevoll R, Menten J, Rutten EH, Thomas DG, et al: A randomized trial on dose-response in radiation therapy of low-grade cerebral glioma: European Organization for Research and Treatment of Cancer (EORTC) Study 22844. Int J Radiat Oncol Biol Phys 36:549-556, 1996

8. Keles GE, Lamborn KR, Berger MS: Low-grade hemispheric gliomas in adults: a critical review of extent of resection as a factor influencing outcome. J Neurosurg 95:735-745, 2001

9. Kesari S, Schiff D, Drappatz J, LaFrankie D, Doherty L, Macklin EA, et al: Phase II study of protracted daily temozolomide for low-grade gliomas in adults. Clin Cancer Res 15:330-337, 2009

10. Kim YH, Nobusawa S, Mittelbronn M, Paulus W, Brokinkel B, Keyvani K, et al: Molecular classification of low-grade diffuse gliomas. Am J Pathol 177:2708-2714, 2010

11. Louis DN, Ohgaki H, Wiestler OD, Cavenee WK, Burger PC, Jouvet A, et al: The 2007 WHO classification of tumours of the central nervous system. Acta Neuropathol 114:97-109, 2007

12. McGirt MJ, Chaichana KL, Attenello FJ, Weingart JD, Than $\mathrm{K}$, Burger PC, et al: Extent of surgical resection is independently associated with survival in patients with hemispheric infiltrating low-grade gliomas. Neurosurgery 63:700-708, 2008

13. Muragaki Y, Iseki H, Maruyama T, Tanaka M, Shinohara C, Suzuki T, et al: Information-guided surgical management of gliomas using low-field-strength intraoperative MRI. Acta Neurochir Suppl 109:67-72, 2011
14. Nitta M, Muragaki Y, Maruyama T, Iseki H, Ikuta S, Konishi Y, et al: Updated therapeutic strategy for adult low-grade glioma stratified by resection and tumor subtype. Neurol Med Chir (Tokyo) 53:447-454, 2013

15. Pace A, Vidiri A, Galiè E, Carosi M, Telera S, Cianciulli $\mathrm{AM}$, et al: Temozolomide chemotherapy for progressive lowgrade glioma: clinical benefits and radiological response. Ann Oncol 14:1722-1726, 2003

16. Pignatti F, van den Bent M, Curran D, Debruyne C, Sylvester R, Therasse P, et al: Prognostic factors for survival in adult patients with cerebral low-grade glioma. J Clin Oncol 20:2076-2084, 2002

17. Pouratian N, Mut M, Jagannathan J, Lopes MB, Shaffrey ME, Schiff D: Low-grade gliomas in older patients: a retrospective analysis of prognostic factors. J Neurooncol 90:341-350, 2008

18. Sanai N, Berger MS: Glioma extent of resection and its impact on patient outcome. Neurosurgery 62:753-766, 2008

19. Schomas DA, Laack NN, Rao RD, Meyer FB, Shaw EG, O'Neill BP, et al: Intracranial low-grade gliomas in adults: 30-year experience with long-term follow-up at Mayo Clinic. Neuro Oncol 11:437-445, 2009

20. Shaw E, Arusell R, Scheithauer B, O'Fallon J, O'Neill B, Dinapoli R, et al: Prospective randomized trial of low- versus high-dose radiation therapy in adults with supratentorial low-grade glioma: initial report of a North Central Cancer Treatment Group/Radiation Therapy Oncology Group/ Eastern Cooperative Oncology Group study. J Clin Oncol 20:2267-2276, 2002

21. Shaw EG, Wang M, Coons SW, Brachman DG, Buckner JC, Stelzer KJ, et al: Randomized trial of radiation therapy plus procarbazine, lomustine, and vincristine chemotherapy for supratentorial adult low-grade glioma: initial results of RTOG 9802. J Clin Oncol 30:3065-3070, 2012

22. van den Bent MJ, Afra D, de Witte O, Ben Hassel M, Schraub S, Hoang-Xuan K, et al: Long-term efficacy of early versus delayed radiotherapy for low-grade astrocytoma and oligodendroglioma in adults: the EORTC 22845 randomised trial. Lancet 366:985-990, 2005

23. van den Bent MJ, Carpentier AF, Brandes AA, Sanson M, Taphoorn MJ, Bernsen HJ, et al: Adjuvant procarbazine, lomustine, and vincristine improves progression-free survival but not overall survival in newly diagnosed anaplastic oligodendrogliomas and oligoastrocytomas: a randomized European Organisation for Research and Treatment of Cancer phase III trial. J Clin Oncol 24:2715-2722, 2006

24. Wakimoto H, Aoyagi M, Nakayama T, Nagashima G, Yamamoto S, Tamaki M, et al: Prognostic significance of Ki-67 labeling indices obtained using MIB-1 monoclonal antibody in patients with supratentorial astrocytomas. Cancer 77:373-380, 1996

25. Youland RS, Brown PD, Giannini C, Parney IF, Uhm JH, Laack NN: Adult low-grade glioma: 19-year experience at a single institution. Am J Clin Oncol 36:612-619, 2013

\section{Author Contributions}

Conception and design: Muragaki, Nitta, Maruyama, Komori, Maebayashi, Iseki, Okada. Acquisition of data: Nitta, Maruyama, Ikuta. Analysis and interpretation of data: Nitta, Ikuta. Drafting the article: Muragaki, Nitta. Critically revising the article: Chernov. Reviewed submitted version of manuscript: Muragaki, Nitta, Maruyama, Komori, Maebayashi, Iseki, Tamura, Saito, Okamoto, Chernov, Hayashi, Okada. Approved the final version of the manuscript on behalf of all authors: Muragaki. Statistical analysis: Nitta, Ikuta. Study supervision: Muragaki, Iseki, Okada.

\section{Correspondence}

Yoshihiro Muragaki, Department of Neurosurgery, Tokyo Women's Medical University Hospital, 8-1 Kawada-cho, Shinjuku-ku, Tokyo 162-8666, Japan. email: ymuragaki@twmu.ac.jp. 